\title{
Nanometer emittance ultralow charge beams from rf photoinjectors
}

\author{
R. K. Li, K. G. Roberts, C. M. Scoby, H. To, and P. Musumeci \\ Department of Physics and Astronomy, UCLA, Los Angeles, California 90095, USA
}

(Received 19 June 2012; published 11 September 2012)

\begin{abstract}
In this paper we discuss the generation of a new class of high brightness relativistic electron beams, characterized by ultralow charge $(0.1-1 \mathrm{pC})$ and ultralow normalized emittance $(<50 \mathrm{~nm})$. These beams are created in rf photoinjectors when the laser is focused on the cathode to very small transverse sizes $(<30 \mu \mathrm{m} \mathrm{rms})$. In this regime, the charge density at the cathode approaches the limit set by the extraction electric field. By shaping the laser pulse to have a cigarlike aspect ratio (the longitudinal dimension much larger than the transverse dimension) and a parabolic temporal profile, the resulting space charge dominated dynamics creates a uniformly filled ellipsoidal distribution and the emittance can be nearly preserved to its thermal value. We also present a new method, based on a variation of the pepper-pot technique, for single shot measurements of the ultralow emittances for this new class of beams.
\end{abstract}

DOI: 10.1103/PhysRevSTAB.15.090702

PACS numbers: 29.25.Bx, 29.27.Bd, 41.75. Ht, 41.85.Ct

\section{INTRODUCTION}

A recent trend in the development of high brightness electron beam sources is lower charge, lower emittance beams. For decades, electron accelerators and sources have been designed aiming at a traditional $1 \mathrm{nC} / 1 \mathrm{~mm} \mathrm{mrad}$ goal dictated by luminosity requirements for high energy physics as well as photon flux in light source applications. Part of this is simply historical. Even as it becomes clear that lower charge beams characterized by smaller emittances and shorter bunch lengths can be very useful for a variety of applications, most of the beam diagnostics developed and installed on existing beam lines are blind at very low charge levels.

As the charge is reduced to $<1 \mathrm{pC}$, which in this paper is considered as the boundary of ultralow charge, the phase space area of the beam can be greatly reduced and "ultralow" normalized transverse emittances of $<0.1 \mathrm{~mm}$ mrad become possible [1]. Here we focus mostly on the transverse phase space, but it should be pointed out that one of the main motivations behind the charge reduction trend is the possibility of greatly shortening the beam bunch length by downstream compression, effectively keeping constant the peak current at the end of the machine. A direct application of ultralow charge ultralow emittance beams is high resolution ultrafast relativistic electron diffraction and microscopy where the beam is used to resolve temporally and spatially small changes in matters [2-4]. Applications of these beams as drivers for sub-fs high gain free-electron laser amplifiers can also be

Published by the American Physical Society under the terms of the Creative Commons Attribution 3.0 License. Further distribution of this work must maintain attribution to the author(s) and the published article's title, journal citation, and DOI. envisioned [5-7]. Low charge beams occupying very small area in phase space are also suitable for external injection into laser-based high gradient acceleration structures [8,9].

So far, one of the highest brightnesses reported from a radio-frequency (rf) photoinjector has been the $0.2 \mathrm{~mm}$ mrad projected emittance $(0.14 \mathrm{~mm}$ mrad slice emittance) obtained with $20 \mathrm{pC}$ at SLAC using the Linac Coherent Light Source (LCLS) injector [10]. This beam, with far lower charge than the original LCLS design working point, has enabled a new regime of operation of the x-ray laser characterized by fs pulses and short free-electron laser gain length. More recently, different groups have reported comparable brightness [11-14]. One should point out here that measurement systematics (in the technique, in the image processing, etc.) could affect significantly the reported brightness values. Care must be taken when comparing different group results (for example, some quote $90 \% \mathrm{rms}$ emittance, others full rms emittance, etc.) [15]. More than focusing on the absolute emittance and brightness values, here we discuss a new approach to the generation of ultralow charge ultralow emittance beams.

In this paper we describe the study of the regime of operation of an rf photoinjector where the laser is focused on the cathode to a very small ( $\lesssim 30 \mu \mathrm{m} \mathrm{rms}$ ) transverse spot. This regime is characterized by a strong space charge driven transverse expansion which, under properly chosen initial conditions, leads to the creation of uniformly filled ellipsoidal beams [16]. The scheme has similarities with the previously demonstrated blow-out regime of rf photoinjectors [17-19] but instead of relying on the longitudinal space charge forces for beam shaping, uses the transverse forces to generate the ellipsoidal distribution. An important difference is that ultralow beam emittances can be obtained due to the minimization of the thermal emittance contribution. 


\section{A SIMPLIFIED ENVELOPE EQUATION MODEL AND SIMULATION}

In order to understand better the process of the generation of the uniformly filled ellipsoidal distribution, we consider the space charge dominated transverse expansion of a beam slice with current $I$ and initial spot size $\sigma_{0}$. The evolution of the rms transverse beam size $\sigma_{x}$ can be estimated using the beam envelope equation. In the space charge dominated regime, we can neglect the emittance contribution term and the equation reads [20]

$$
\sigma_{x}^{\prime \prime}+k_{\beta}^{2} \sigma_{x}=\frac{I}{2 I_{A} \gamma^{3} \sigma_{x}},
$$

where $I_{A}=17 \mathrm{kA}$ is the Alfven current and $k_{\beta}^{2}$ represents the focusing system. This equation describes the transverse size of each longitudinal slice within the beam if laminar flow conditions apply. The equilibrium condition (Brillouin flow) consists in a transverse size which scales as the $I(\zeta)^{1 / 2}$, where we added the $\zeta$ dependence to stress the fact that each slice can have a different current and hence a different equilibrium transverse size. In an rf photoinjector beam line, the situation is more complicated than the simple case discussed here; since the beam energy is changing, there is the effect of the rf fields, and the focusing channel (emittance compensation solenoid) is not uniform along the beam propagation. On the other hand, it can be verified by particle tracking simulations that the scaling of the output transverse size on the beam current remains similar so that to a good approximation at the exit of the photoinjector we have $\sigma_{x}(\zeta) \propto I(\zeta)^{1 / 2}$. This is an important result since it implies that the final current density $J(\zeta) \propto I(\zeta) / \sigma_{x}(\zeta)^{2}$ will be independent of the longitudinal coordinate $\zeta$ along the bunch.

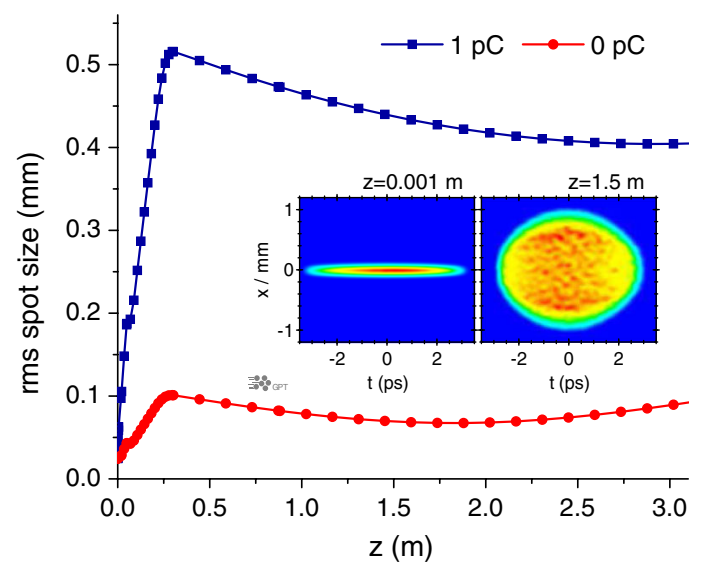

FIG. 1. GPT simulations of cigar beam dynamics in rf photoinjectors: transverse beam sizes along the Pegasus beam line for $1 \mathrm{pC}$ and $0 \mathrm{pC}$ (space charge effects switched off). The simulation parameters match the experimental ones reported in Table I. With proper initial conditions, the space charge dominated expansion leads to the creation of a nearly ideal uniformly filled ellipsoidal distribution (inset).
By choosing the input current profile (shaping the temporal profile of the laser pulse on the cathode) to be parabolic $I(\zeta)=I_{0}\left[1-(\zeta / L)^{2}\right]$ where $L$ is a characteristic dimension of the bunch length (the rms bunch length $L / \sqrt{5}$ ), the beam edge will be well described by the curve $\sqrt{1-(\zeta / L)^{2}}$ which is the equation of an ellipse. General Particle Tracer (GPT) [21] simulations confirm that, when the beam dynamics is completely dominated by the space charge forces, a strong transverse expansion takes place and a cigarlike shaped beam with a parabolic current profile does evolve into a nearly ideal uniformly filled ellipsoidal distribution (see Fig. 1).

\section{CREATION OF CIGARLIKE SHAPED UNIFORMLY FILLED ELLIPSOID BEAMS}

The experiment took place at the UCLA Pegasus rf photoinjector beam line. The details of the experimental setup have been described elsewhere [19]. We summarize the parameters used for this experiment in Table I. A bare (no coating) copper cathode was installed in the gun at the time of these experiments and $266 \mathrm{~nm}$ laser was used to generate the beam by linear photoemission. A $1.5 \mathrm{~m}$ focal distance lens was used to focus the laser pulse on the cathode to a spot size of $\sigma_{0}=30 \mu \mathrm{m} \mathrm{rms}$ as verified by transverse laser profile measurements performed with a CCD camera at the cathode plane. A UV stretcher with adjustable dispersion was used to control the chirp of the laser pulse and modify its length. The UV laser spectrum as measured by an Ocean Optics spectrometer was with a good approximation parabolic. The temporal profile of the stretched UV pulse had the same shape. The electric field of the gun was maintained low $(\sim 70 \mathrm{MV} / \mathrm{m})$ to reduce the dark current and allow direct measurements of the photoelectron beam properties. The rf electric field at injection sets a limit on the amount of charge that can be extracted from the cathode, which is still bounded by the threshold for virtual cathode formation as indicated by Bazarov et al. [1]. For 30 degrees injection phase the charge is limited to $\sim 1 \mathrm{pC}\left(Q / \epsilon_{0} 2 \pi \sigma_{0}^{2} \cong 20 \mathrm{MV} / \mathrm{m}\right)$. If one could increase the field in the gun (possibly jointly with proper copper surface treatment to maintain the dark current to a level compatible with low charge beam diagnostics), it would be possible to extract more charge from

TABLE I. Pegasus photoinjector parameters for the transverse space charge dominated expansion regime.

\begin{tabular}{lc}
\hline \hline Beam total energy & $3.5 \mathrm{MeV}$ \\
Peak field at the cathode & $70 \mathrm{MV} / \mathrm{m}$ \\
Injection phase & $30 \mathrm{deg}$ \\
Beam charge & $1 \mathrm{pC}$ \\
Laser spot size (rms) & $30 \mu \mathrm{m}$ \\
Laser pulse length (rms) & $1.8 \mathrm{ps}$ \\
Bunch length at rf deflector (rms) & $1.6 \mathrm{ps}$ \\
Normalized emittance & $40 \mathrm{~nm}$ \\
\hline \hline
\end{tabular}


the same cathode area and generate a higher brightness beam. Another issue when focusing the laser on the cathode to a very small spot is related to the possible damage to the cathode surface. The maximum laser intensity in these experiments was a few $\mathrm{GW} / \mathrm{cm}^{2}$ (assuming $1-2 \mu \mathrm{J}$ in $5 \mathrm{ps}$ FWHM), similar to what was used in the blow-out experiment and significantly below the damage threshold for a flat copper surface (a few hundred $\mathrm{GW} / \mathrm{cm}^{2}$ ).

In Fig. 2(a) we show the transverse beam profile of the cigarlike beam measured at a fluorescent screen located $3.0 \mathrm{~m}$ downstream of the cathode, together with the GPT simulation results in Fig. 2(b). The point spread function of the screen and imaging system was $65 \mu \mathrm{m} \mathrm{rms}$. One should notice how clean and round the beam profile looks. Laser and cathode inhomogeneities which usually affect and distort the shape of the electron beam are essentially absent. In this regime, the beam profile is completely determined by the strong space charge dominated transverse expansion and no feature from the initial spatial distribution survives after the gun exit. The projection of the beam profile onto one axis (dashed line) is well fitted by a parabolic distribution (solid line), as expected for a uniformly filled ellipsoidal beam.

The transverse profile of the beam when the UV stretcher was off [measurement and simulation results in Figs. 2(c) and 2(d), respectively] shows a bright corona, indicating the onset of wave breaking typical of nonlinear space charge forces. When the beam is short at the cathode there is a strong coupling between transverse and longitudinal expansions and our simplified model for the beam evolution no longer applies. The folding of the beam in phase space causes irreversible emittance growth and
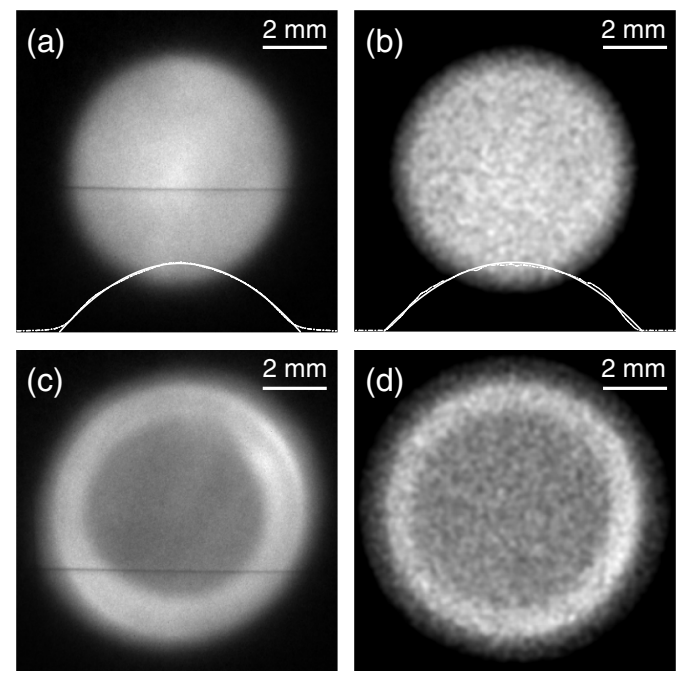

FIG. 2. Measured transverse beam profiles at $z=3.0 \mathrm{~m}$ from the cathode for (a) a stretched laser pulse and (c) a short laser pulse on the cathode. Corresponding GPT simulation results are shown in (b) and (d). The projection of the beam profiles in (a) and (b) onto one axis (dashed line) are also shown together with the parabolic fitting (solid line). degrades the beam quality. GPT simulations closely reproduce this dynamical behavior. In Fig. 3(a) we show the simulated transverse phase space $x-\beta_{x}$ (red dot) and the density profile along the $x$ axis (blue line) at $z=$ $3 \mathrm{~m}$. The distribution is "narrow" in the central part and becomes "wider" toward both ends where the wave breaking occurs. The correlation between the transverse positions of the particles at the cathode $r_{i}$ and their positions $r_{f}$ on the screen displayed in Fig. 3(b) clearly shows the loss of laminarity due to the space charge driven wave breaking. Incidentally, it is interesting to notice that the central part of the beam which samples only the linear component of the space charge forces shows a local maximum in phase space density. Using a collimating hole it could be possible to select this high brightness region of the beam and utilize it for some applications that demand very low charge beams such as ultrafast relativistic electron diffraction and microscopy.

Thanks to the nearly perfect shape of the beam, it becomes easier to monitor (and compensate if needed) quadrupole components (normal and skew) along the beam line. We will come back to this point towards the end of the paper.

In order to verify that the longitudinal profile of the cigarlike beam is also parabolic, we used the $9.6 \mathrm{GHz}$ $500 \mathrm{kV}$ X-band deflecting cavity installed on the Pegasus beam line [22] and imaged the beam in the $x-z$ plane, as shown in Fig. 4(a). Its projection onto the $z(t)$ axis (red dot) shown in Fig. 4(c) well resembles a parabolic profile except the tails at the beam edges which are due to the finite transverse spot sizes on the screen. As a comparison, an ideal 1.8 ps rms parabolic UV laser pulse was input into GPT simulation and the result is shown (black solid line) after convoluted with the transverse spot size. Conversely, if the laser pulse on the cathode is short, the beam expansion is strongly asymmetrical due to the strong image charge effect. This is confirmed by the measurement [Fig. 4(a) and blue square in (c)] with the deflector when
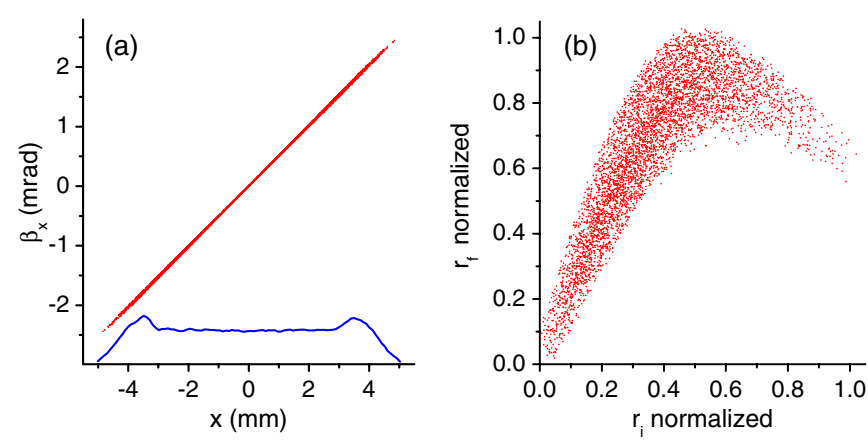

FIG. 3. (a) The transverse phase space $x-\beta_{x}$ of the beam when the UV stretcher was off and the laser pulse short at the cathode. The density profile along $x$ axis is also shown (blue line). (b) The correlation between the initial transverse positions $r_{i}$ at the cathode and the final positions $r_{f}$ on the screen. 
(a) with stretched (1.8 ps rms) UV laser pulse

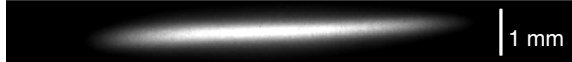

(b) with short (65 fs rms) UV laser pulse

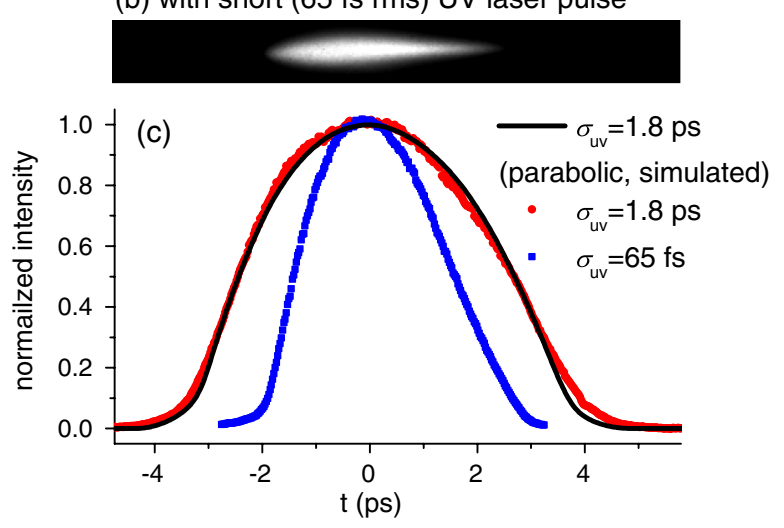

FIG. 4. Beam profiles on a transverse screen after streaked by an rf deflecting cavity when the UV laser pulse is (a) stretched to $1.8 \mathrm{ps} \mathrm{rms}$, and (b) an ultrashort $65 \mathrm{fs}$ rms distribution. Their projections onto the $t(z)$ axis are shown in (c) together with the GPT simulation result (black solid line) for an ideal $1.8 \mathrm{ps} \mathrm{rms}$ parabolic UV laser pulse.

the UV stretched is bypassed and the laser pulse length on the cathode is $65 \mathrm{fs}$ rms. Longitudinal laser profile shaping is required in order to create a distribution that is a good approximation of a uniformly filled ellipsoidal distribution.

\section{MEASUREMENT OF ULTRALOW EMITTANCE}

Simulations predict sub-100 nm normalized emittances for the beams created in this regime, due to the very small thermal emittance contribution from the small laser spot on the cathode. It remains a challenge to measure the sub$100 \mathrm{~nm}$ normalized emittance of this new class of ultralow charge beams. The pepper-pot technique [23] is a natural candidate to sample in a single shot the divergence at multiple transverse positions within the beam. However, to apply this method to simultaneously resolve the very small spot size and low divergence with a limited number of electrons, it demands a revision of the beam optics, the pepper-pot design, as well as the detection efficiency and spatial resolution of the beam profile monitor.

First of all, higher detection efficiency with high spatial resolution in imaging the beam profile is demanded. This has been demonstrated recently in works motivated by the requirement of ultrafast relativistic electron diffraction experiments [24]. The new high efficiency detector system developed at UCLA Pegasus Laboratory finds its immediate application also in beam halo mapping and ultralow charge beam emittance measurement which is the topic of this paper.

To simultaneously resolve the very small spot size and very low divergence, we developed a variation of the pepper-pot technique in this work. Instead of a customized pepper-pot target manufactured by laser drilling a regular hole array pattern on a high density metal sheet, we chose commercially available transmission electron microscopy (TEM) grids as the sampling target. For example, a 200 mesh (pitch size $d=125 \mu \mathrm{m}$ ) copper grid (G200TH from SPI, Inc.), as shown in Figs. 5(b) and 5(c), was chosen for the data presented in this paper. It consists of perpendicular copper bars that are $32 \mu \mathrm{m}$ wide and $20 \pm 3 \mu \mathrm{m}$ in thickness.

Interestingly, TEM grids have also been used to indicate the beam quality in electron microscopy community by observing the Fresnel fringes formed on the edge of the shadow image of the grid bar [25]. As the electrons in the beam propagate through the grid, those hitting the bars will be scattered into a broad angular distribution and the transmitted ones form a shadow image of the grid. Imagining an electron beam with a zero emittance, i.e. all the electrons originate from one point before the grid, the shadow image will have a sharp transition across the edge of the grid bar. For a real beam, the edge in the image will smear due to the finite beam divergence. By analyzing the position, width, and height of each edge in the shadow image, we can reconstruct the trace space and retrieve the emittance value of the beam at the grid position.

The relative thin TEM grid bars are not able to block the relativistic electrons and one concern is if the scattered electrons contaminate the phase space sampling and emittance measurement. Roughly $50 \%$ of the electrons in the beam pass through the grid openings and are measured by the screen $2.1 \mathrm{~m}$ after the grid. All the other electrons that hit the copper bars, according to Monte Carlo simulation results, are scattered into a broad angular distribution and only $<0.1 \%$ will be in a cone within $2.5 \mathrm{mrad}$ from the beam line axis and overlap with the transmitted beam whose full transverse size on the screen is smaller than $10 \mathrm{~mm}$. This is actually not surprising since the $20 \mu \mathrm{m}$ thickness is already $\sim 300$ times the elastic mean-free path. It is safe to conclude that the grid is thick enough to perform hard-edge sampling of the beam trace space.
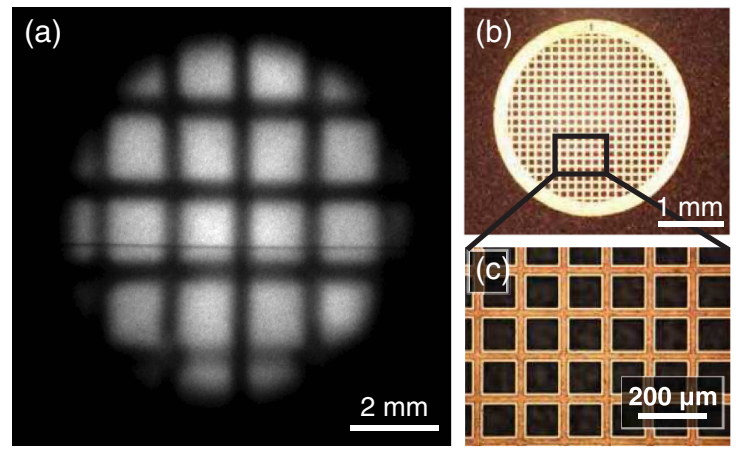

FIG. 5. (a) Electron beam shadow image on the detector screen $2.1 \mathrm{~m}$ after the TEM grid. The image of the grid appears magnified by a factor of 12.8. (b), (c) Optical microscope images of the 200 mesh TEM grid. 
A typical shadow image of the TEM grid is shown in Fig. 5(a). The strength of the emittance compensation solenoid is tuned to deliver a sharp beam focus at $L_{0} \sim$ $20 \mathrm{~cm}$ before the grid. Similar to the pepper-pot technique in which the size of each beamlet image gives a measure of the divergence, in this grid method information on the local beam divergence can be extracted by analyzing the image profile at each bar edge. However, there is a considerable difference in the data processing technique. The pepper-pot method had been applied to larger emittance beams and the divergence-induced increase in beamlet image is usually much larger than the projection of the hole itself. For the ultralow emittance case, the ratio between the two contributing terms can be very different.

Consider the trace space of the beam right after a TEM grid, as illustrated in Fig. 6(a), for two adjacent transmitted beamlets assuming they are uniform in density and Gaussian in divergence with an rms width of $\sigma_{x^{\prime}}$. After a free space drift distance of $L$ from the grid to the screen, the beam trace space is sheared as shown in Fig. 6(b) and the intensity profile measured by the screen, displayed in the inset of Fig. 6(b), can be fitted by

$$
g(x) \propto \operatorname{erf}\left(\frac{x+M a / 2}{\sqrt{2} L \sigma_{x^{\prime}}}\right)-\operatorname{erf}\left(\frac{x-M a / 2}{\sqrt{2} L \sigma_{x^{\prime}}}\right),
$$

where $a$ is the bar width and $M=1+L / L_{0}$ is the magnification of the shadow image. One may notice that in the limit of $M a \ll L \sigma_{x^{\prime}}$, the above expression reduces to the Gaussian distribution typically used for emittance measurement with pepper-pot or multislit techniques. In order to minimize the overlap of the signal from two nearby edges, it is important to pick the TEM grid and the experimental parameters so that $M a \gtrsim 4 L \sigma_{x^{\prime}}$. This is not a
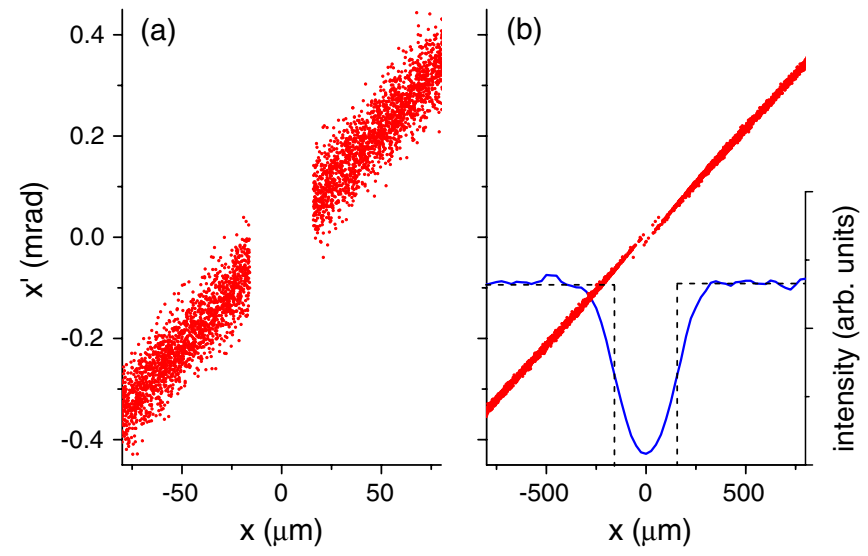

FIG. 6. Zoomed in snapshots of the $x-x^{\prime}$ trace space of the beam (a) just blocked by a $32 \mu \mathrm{m}$ grid bar and (b) at the beam profile screen $2.1 \mathrm{~m}$ after the grid. In (a) the beam has a uniform density along $x$ and a Gaussian distribution in $x^{\prime}$ with an rms size of $40 \mu \mathrm{rad}$. The slope of the $x-x^{\prime}$ band magnifies the bar image by a factor of 10 . In the inset of (b) we show the shadow image of the grid bar by an ideal point source (dashed black) and the density profile of a realistic beam (solid blue). stringent requirement as the fitting of the measured profile can tolerate a slight overlap as seen in Fig. 6(b). In all of the commercial TEM grids, the grid opening $d-a$ is larger than the bar width $a$ so that cross talking between two adjacent bars is avoided. The separation between the two edges can be increased by increasing $M$ however at a price that the beam spot gets smaller at the grid and the trace space sampling is too coarse. In order to resolve small beam divergence, it is mandatory to optimize the spatial resolution of the beam profile detector. In practice this can be quantified by requiring that the size of the point spread function $\sigma_{\text {psf }}$ should be much smaller than $L \sigma_{x^{\prime}}$. In our measurements we ensured that $L \sigma_{x^{\prime}}$ was typically at least 2 times as large as $\sigma_{\mathrm{psf}}$ and then removed its contribution through deconvolution.

The TEM grid method is in principle analogous to the pepper-pot technique but has the advantage that a much larger portion of electrons is transmitted, which for ultralow charge beams improves the signal-to-noise ratio of the shadow image and allows the trace space sampling to achieve a higher precision. Besides extracting two separate emittance values for the $x$ and $y$ planes, the shadow image also contains the degree of correlation between $x$ and $y$ directions. For example, the deviation from $90^{\circ}$ of the crossing bars is an indication of the $x-p_{y}$ correlation (angular momentum) of the beam which is usually induced by a skew quadrupole field component inside the emittance compensation solenoid. Thus, the shadow image provides an intuitive guide for online error field correction and emittance optimization.

The results of the emittance measurements in the cigar beam operating mode are shown in Fig. 7 for the cases of long and short laser pulses on the cathode. After correcting any skew quadrupole error the emittance numbers for $x$ and $y$ planes are within $10 \%$ and each data point represents $\sqrt{\epsilon_{n x} \epsilon_{n y}}$. The measurement and simulation results agree

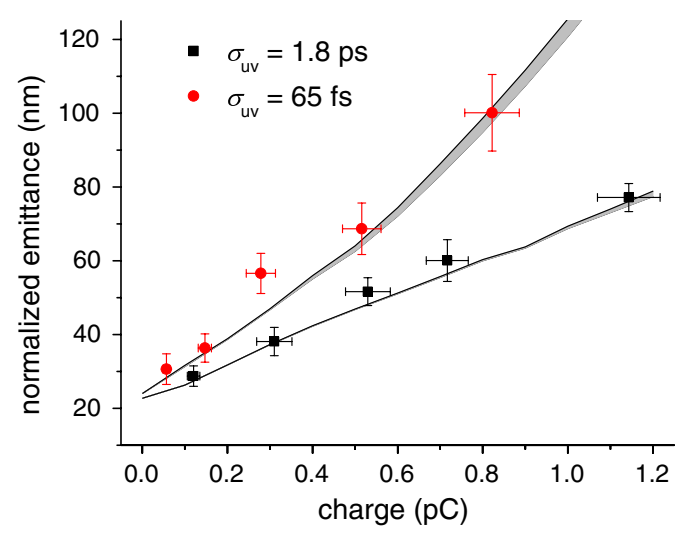

FIG. 7. Measured normalized emittance of beams when the UV laser was stretched to a $1.8 \mathrm{ps}$ rms parabolic longitudinal profile (black square) and was ultrashort at $65 \mathrm{fs} \mathrm{rms} \mathrm{(red} \mathrm{circle).}$ The error bars indicate the statistics over 20 shots. Simulation results for both cases are also shown. 
well for both cases throughout the entire charge range. Particularly, the measured emittance numbers converge toward a same thermal emittance value at low charge. For both the long and short laser cases, the laser spot sizes on the cathode were $30 \mu \mathrm{m} \mathrm{rms}$ and the measurement indicates a thermal emittance level of $0.8 \mathrm{~mm} \mathrm{mrad} / \mathrm{mm}$ rms which is consistent (within 20\%) with the results from other groups [10,26,27].

We would like to emphasize that the emittance values reported in Fig. 7 do not correspond to the minimum emittances that could be achieved when operating the photoinjector in the cigar beam mode. This is due to the limitation of the Pegasus beam line that features only one strong focusing element, the gun solenoid, which was used for the measurements to provide a sharp focus before the TEM grid. GPT simulations predict that lower emittances could be obtained with relaxed focusing condition due to partial emittance compensation (realignment of the slices in trace space) [28-30]. The comparison of the "optimal emittance mode" and the "emittance measurement mode" with different solenoid strengths is shown in Fig. 8 with the parameters listed in Table I. A possibility for future investigation of this regime would be to extend the Pegasus beam line and insert another strong focusing element to measure the $1 \mathrm{pC}$ beam at the location of its minimum emittance which is as low as $40 \mathrm{~nm}$.

Although the beam charge discussed is ultralow, due to the small spot size and low emittance values the beam may still be space charge dominated and the space charge effects may play a role in the emittance measurement. In the emittance measurement mode at the grid position, the parameter $R_{0}=I \sigma_{0}^{2} /\left(2 I_{0} \gamma \epsilon_{n}^{2}\right)$, which is used to characterize the relative importance of space charge effects and emittance pressure [31], is on the order of 1 for $1 \mathrm{pC}$ beams. Nevertheless, due to the strong focusing before

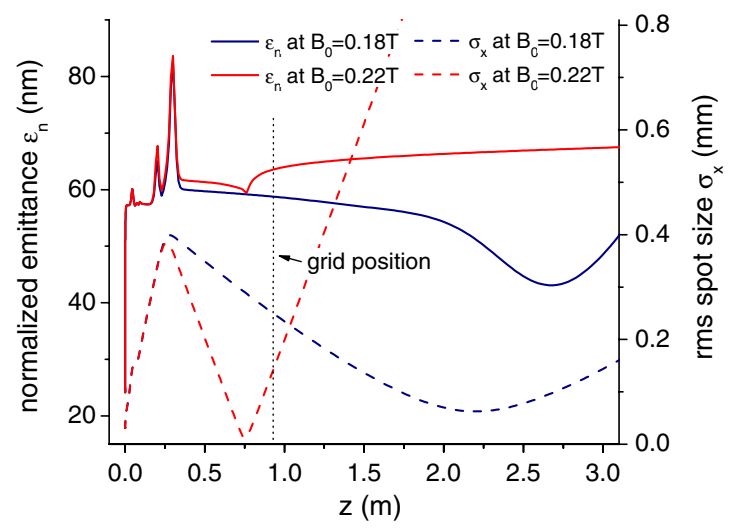

FIG. 8. Comparison of the emittance (solid lines) and spot size (dashed lines) along the beam line for the "optimal emittance mode" $\left(B_{0}=0.18 \mathrm{~T}\right)$ and the "emittance measurement mode" $\left(B_{0}=0.22 \mathrm{~T}\right)$. The solenoid strength is tuned to deliver the minimum emittance in the first mode and focus strongly to provide suitable $M$ for emittance measurement using the grid method in the later mode, respectively. the grid, the beam size quickly expands and the $R_{0}$ value decreases by a factor of 4 in every $\sim 20 \mathrm{~cm}$. The overall effect is that the space charge induced distortion of the trace space between the grid and screen has negligible effects on the measured emittance values. This is quantitatively verified by testing the data processing algorithm through GPT simulations. The agreement is within $\pm 10 \%$ for the $0.1-1 \mathrm{pC}$ and $30-100 \mathrm{~nm}$ range.

\section{OUTLOOK AND CONCLUSIONS}

In this paper we explored for the first time the cigar beam operating mode in an S-band rf photoinjector. The transverse and longitudinal beam profiles were measured and found in a good approximation to be parabolic profiles, consistent with being the projection of a uniformly filled ellipsoidal distribution. The simulations as well as the measurements reveal residual nonlinearities in the phase space (manifested in space charge induced emittance growth), due to the fact that the equilibrium solution is not fulfilled from its generation. The beams in fact always first goes through a "pancake" regime very close to the cathode when the velocities are much smaller than the speed of light. In order to improve the beam brightness, a more careful control of the initial distribution (laser shape) is required. A plot of normalized emittance from GPT simulation is shown in Fig. 9 for three different initial transverse profiles (uniform, spherical, and Gaussian). The gun gradient is boosted to $120 \mathrm{MV} / \mathrm{m}$ thus allowing $1 \mathrm{pC}$ charge to be extracted from a smaller cathode area of $20 \mu \mathrm{m} \mathrm{rms}$. The longitudinal laser profile as well as the other parameters in the system, such as solenoid strength and injection phase etc., are same for all cases. With a

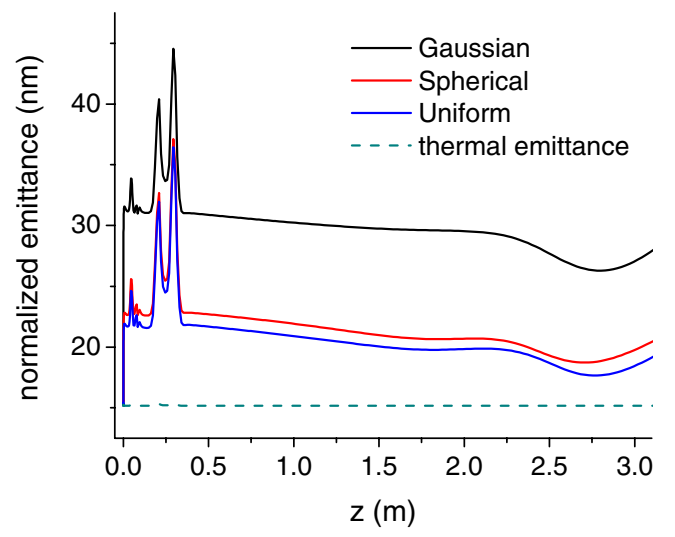

FIG. 9. Comparison of the optimal emittance for Gaussian, spherical, and uniform initial transverse distributions from the cathode (all have $20 \mu \mathrm{m} \mathrm{rms} \mathrm{spot} \mathrm{sizes)} \mathrm{for} \mathrm{a} \mathrm{gun} \mathrm{gradient} \mathrm{of}$ $120 \mathrm{MV} / \mathrm{m}$. For the Gaussian profile the nonlinear transverse space charge forces at the beam edge lead to slice emittance growth. For spherical and uniform cases the transverse space charge forces are more linear and the compensated projected emittance is closer to the thermal emittance level. In the later cases $1 \mathrm{pC}$, sub-20 $\mathrm{nm}$ beams are generated. 
Gaussian profile, which is the best approximation to the experimental laser profile used for the measurements in this paper, the nonlinear space charge forces at the beam edge leads to non-negligible slice emittance growth. A new laser transport and transverse shaping configuration needs to be designed in order to provide a uniform, ultrasmall spot on the cathode. This proves to be a technical challenge in our setup due to the long distance between the last lens on the normal incidence optical line and the cathode $(150 \mathrm{~cm})$ so that diffraction effects become noticeable. Laser illumination from the oblique window on the half cell of the gun is a possible solution. A new rf gun cathode with back illumination is another very interesting route.

We also discussed here a new emittance measurement technique to measure the ultralow $(30-100 \mathrm{~nm})$ normalized emittance of this new class of ultralow charge $(0.1-1 \mathrm{pC})$ beams. It is based on a variation of the pepper-pot method but using cheap commercially available TEM grids as the sampling target, focusing the beam very hard before the grid to form a magnified shadow image on a downstream screen. The divergence at the bar edges and the beam profile can be extracted with an erf function model. There is good consistency between the measurement results and simulation predictions demonstrating the validity of the new method. In the current work the main solenoid was used to strongly focus the beam for emittance measurement instead of emittance compensation; thus, the emittance numbers were not the best achievable values. In the future at Pegasus or other beam lines, a strong permanent magnet quadrupole and a TEM grid could be inserted at the minimum emittance position or any other positions for independent emittance diagnostics. We would like to point out the promising potential of this method to measure sub-nm geometric emittance. For example, we choose a 2000 mesh grid $(5 \mu \mathrm{m}$ bar width and $12.5 \mu \mathrm{m}$ pitch size) and focus the beam $2.5 \mathrm{~cm}$ before the grid. At the grid position the beam will cover 4-5 pitches allowing adequately fine sampling and on a screen $3.0 \mathrm{~m}$ after the grid the images of the bar edges have a characteristic size of $L \sigma_{x^{\prime}}$ typically larger than $60 \mu \mathrm{m}$. A phosphor screen and imaging system with enough efficiency and $<20 \mu \mathrm{m}$ rms point spread function is easily achievable and enough to resolve the beam divergence. Such a beam has a geometric emittance of $0.3 \mathrm{~nm}$ and a normalized emittance of $2.2 \mathrm{~nm}$ at $3.5 \mathrm{MeV}$.

In summary, we have studied experimentally a new regime of operation of $\mathrm{rf}$ photoinjectors where the electron beam generated by a narrowly focused, longitudinally shaped laser pulse evolves into a nearly ideal uniformly filled 3D ellipsoid. This regime is complementary to the "blow-out" case but features very small transverse emittance and due to the limitations imposed by the extraction field at the cathode is characterized by very low charge beams. If postacceleration rf structures or magnetic chicanes are available, one has the possibility to compress the beam to achieve very high brightness. The ultralow charge ultralow emittance regime described in this paper could benefit many future researches and applications including single-spike advanced light sources, ultrafast electron diffraction and microscopy, and external injection for wakefield accelerations.

\section{ACKNOWLEDGMENTS}

This work was supported by DOE Grants No. DE-FG0292ER40693 and No. DEFG02-07ER46272 and ONR Grant No. N000140711174.

[1] I. V. Bazarov, B. M. Dunham, and C. K. Sinclair, Phys. Rev. Lett. 102, 104801 (2009).

[2] X. J. Wang, Z. Wu, and H. Ihee, in Proceedings of the 20th Particle Accelerator Conference, Portland, OR, 2003 (IEEE, New York, 2003), WOAC003.

[3] J. B. Hastings, F. M. Rudakov, D. H. Dowell, J. F. Schmerge, J. D. Cardoza, J. M. Castro, S. M. Gierman, H. Loos, and P. M. Weber, Appl. Phys. Lett. 89, 184109 (2006).

[4] P. Musumeci, J. T. Moody, C. M. Scoby, M. S. Gutierrez, and M. Westfall, Appl. Phys. Lett. 97, 063502 (2010).

[5] J. B. Rosenzweig et al., Nucl. Instrum. Methods Phys. Res., Sect. A 593, 39 (2008).

[6] S. Reiche, P. Musumeci, C. Pellegrini, and J.B. Rosenzweig, Nucl. Instrum. Methods Phys. Res., Sect. A 593, 45 (2008).

[7] M. Boscolo, M. Ferrario, I. Boscolo, F. Castelli, S. Cialdi, V. Petrillo, R. Bonifacio, L. Palumbo, and L. Serafini, Nucl. Instrum. Methods Phys. Res., Sect. A 593, 137 (2008).

[8] X. F. D. Stragier, O. J. Luiten, S. B. van der Geer, M. J. van der Wiel, and G.J.H. Brussaard, J. Appl. Phys. 110, 024910 (2011).

[9] A. R. Rossi et al., in Proceedings of the 3rd International Particle Accelerator Conference, New Orleans, LA, 2012 (IEEE, New York, 2012), WEEPPB002.

[10] Y. Ding et al., Phys. Rev. Lett. 102, 254801 (2009).

[11] F. Stephan et al., Phys. Rev. ST Accel. Beams 13, 020704 (2010).

[12] M. Ferrario et al., Phys. Rev. Lett. 104, 054801 (2010).

[13] B. Dunham et al., in Proceedings of the 3rd International Particle Accelerator Conference, New Orleans, LA, 2012 (IEEE, New York, 2012), MOOAA01.

[14] N. Terunuma et al., Nucl. Instrum. Methods Phys. Res., Sect. A 613, 1 (2010).

[15] Mini Workshop on Characterization of High Brightness Beams, DESY, Zeuthen, Germany, 2008 [http://wwwzeuthen.desy.de/chbb08/].

[16] B. J. Claessens, S.B. van der Geer, G. Taban, E. J.D. Vredenbregt, and O. J. Luiten, Phys. Rev. Lett. 95, 164801 (2005).

[17] L. Serafini, AIP Conf. Proc. 413, 321 (1997).

[18] O. J. Luiten, S. B. van der Geer, M. J. de Loos, F. B. Kiewiet, and M. J. van der Wiel, Phys. Rev. Lett. 93, 094802 (2004).

[19] P. Musumeci, J. T. Moody, R. J. England, J. B. Rosenzweig, and T. Tran, Phys. Rev. Lett. 100, 244801 (2008). 
[20] M. Reiser, Theory and Design of Charged Particle Beams (Wiley, New York, 1994).

[21] The General Particle Tracer [http://www.pulsar.nl/gpt/].

[22] J.T. Moody, P. Musumeci, M.S. Gutierrez, J.B. Rosenzweig, and C.M. Scoby, Phys. Rev. ST Accel. Beams 12, 070704 (2009).

[23] M. Zhang, Report No. FERMILAB-TM-1988, 1996.

[24] R. K. Li, P. Musumeci, H. A. Bender, N. S. Wilcox, and M. Wu, J. Appl. Phys. 110, 074512 (2011).

[25] H. Boersch, Phys. Z. 44, 202 (1943).

[26] C. P. Hauri et al., Phys. Rev. Lett. 104, 234802 (2010).
[27] H. J. Qian, C. Li, Y.C. Du, L.X. Yan, J.F. Hua, W. H. Huang, and C.X. Tang, Phys. Rev. ST Accel. Beams 15, 040102 (2012).

[28] B. E. Carlsten, Nucl. Instrum. Methods Phys. Res., Sect. A 285, 313 (1989).

[29] X. Qiu, K. Batchelor, I. Ben-Zvi, and X. J. Wang, Phys. Rev. Lett. 76, 3723 (1996).

[30] L. Serafini and J. B. Rosenzweig, Phys. Rev. E 55, 7565 (1997).

[31] S. G. Anderson, J. B. Rosenzweig, G. P. LeSage, and J. K. Crane, Phys. Rev. ST Accel. Beams 5, 014201 (2002). 